\title{
Autoconcepto físico en función de variables sociodemográficas y su relación con la actividad física
}

\author{
Physical self-concept in terms of sociodemographic variables \\ and their relationship with physical activity
}

\author{
Manuel Fernández Guerrero, Sebastián Feu Molina, Miriam Suárez Ramírez \\ Facultad de Educación. Universidad de Extremadura. España. \\ CORRESPONDENCIA: \\ Manuel Fernández Guerrero \\ mfernandezg@unex.es \\ Recepción: noviembre 2018• Aceptación: mayo 2019 \\ CÓMO CITAR EL ARTíCULO: \\ Fernández-Guerrero, M., Feu-Molina, S., \& Suárez-Ramírez, M. \\ (2020). Autoconcepto físico en función de variables sociodemo- \\ gráficas y su relación con la actividad física. Cultura, Ciencia y \\ Deporte, 15(44), 189-199.
}

\section{Resumen}

El objetivo de este trabajo es estudiar cómo incide la práctica de actividad física del alumnado de Educación Primaria y Educación Secundaria obligatoria en el autoconcepto físico. Se ha realizado un estudio de carácter transversal, descriptivo e inferencial en el que han participado 1093 estudiantes de la ciudad de Badajoz. Se ha tenido en cuenta variables sociodemográficas y contextuales de los centros educativos entre las que destacan el sexo, la edad, curso académico, carácter del centro y tipo de educación que ofrece. El instrumento utilizado para conocer el autoconcepto es el Cuestionario de Autoconcepto Físico (CAF) de Goñi, Ruiz de Azúa \& Liberal (2004) y el utilizado para conocer el nivel de actividad física es el Cuestionario PAQ-C (Physical Activity Questionnaire for Children) de Kowalski, Crocker y Faulkner (1997). Los resultados de esta investigación muestran cómo la práctica de actividad física se relaciona positivamente con una mejora del Autoconcepto Físico ( $p<$ .05). Por otro lado, el autoconcepto físico es superior en los estudiantes del sexo masculino y a medida que los escolares tienen más edad obtienen puntuaciones más altas de autoconcepto físico.

Palabras claves: Competencia física, fuerza, escolares, educación obligatoria, ejercicio físico.

\begin{abstract}
The objective of this work is to study how the practice of physical activity of the students of Primary Education and Compulsory Secondary Education affects the physical self-concept. A transversal, descriptive and inferential study was carried out in which 1093 students from the city of Badajoz participated. Sociodemographic and contextual variables of educational centers have been taken into account, including sex, age, academic year, character of the center and type of education offered. The instrument used to know the self-concept is the Questionnaire of Physical Self-Concept (CAF) of Goñi, Ruiz de Azúa and Liberal (2004) and the one used to know the level of physical activity is the Questionnaire PAQ-C (Physical Activity Questionnaire for Children) of Kowalski, Crocker and Faulkner (1997). The results of this investigation show how the practice of physical activity is positively related to an improvement of the Physical Self-concept $(p<.05)$. On the other hand, physical selfconcept is higher in male students and as older students get higher scores of physical self-concept.
\end{abstract}

Key words: Perceived competence, strength, students, compulsory education and physical exercise. 


\section{Introducción}

El autoconcepto físico ha sido analizado y abordado por multitud de profesionales (Barrios, Gómez \& Barriopedro, 2017; Esnaola, 2005; Fox, 1990; Garaigordobil, Pérez \& Mozaz, 2008; González \& Fernández, 2017; Goñi \& infante, 2010; Goñi, Ruiz de Azúa \& Liberal, 2004; Goñi \& Zulaika, 2000; Infante \& Goñi, 2009; Infante \& Zulaika, 2008; Klomsten, Skaalvik \& Espnes, 2004; Marsh, 1998; Pérez, García, \& Ferriol, 2015; Rangel, Mayorga, Peinado \& Barrón, 2017).

En los años 90 aparecieron diferentes modelos del autoconcepto físico (Burns, 1990; Fox, 1990; Thompson, Penner \& Atabe, 1990) los cuales sirvieron como base paro los creados en la última década (Esnaola, 2005; Esnaola, Infante, \& Zulaika, 2011) que los dividen en habilidad física, condición física, atractivo físico y fuerza. Dentro de dicha jerarquía se podía observar como resultante dos escalas generales denominadas autoconcepto físico general y autoconcepto general.

El autoconcepto físico es aquella representación mental, física y psicológica que todo individuo tiene de su cuerpo que varía en función del nivel de actividad física, condición física, habilidad y fuerza. Autores como Esnaola, Rodríguez \& Goñi (2011) la definen como el conjunto de percepciones que tienen los individuos de sus habilidades y apariencia física. El termino de autoconcepto siempre ha estado asociado al concepto de autoestima, por ello autores como Topcu, Orthon, Tayfun, Ucaktuerk \& Demirel (2016) tratan de clarificar el término autoconcepto en su diferenciación con la autoestima, relacionándose aspectos evaluativos y afectivos.

El término de autoconcepto físico está ligado al concepto de práctica de actividad física (Alvariñas \& González, 2004; Blanco, Benavides, Tristán \& Mayorga-Vega, 2017; Candel, Olmedilla \& Blas, 2008; Contreras, Fernández, García, Palou \& Ponseti, 2010; Espejo, Zurita, Chacón, Castro, Martínez-Martínez \& Pérez-Cortés, 2018; Fraguela-Vale, Varela-Garrote \& Sanz-Arazuri, 2016; Gálvez, Rodríguez-García, Rosa, García-Cantó, Pérez-Soto, Tárraga \& Tárraga, 2016; Goñi, Esnaola, Rodríguez \& Camino, 2015; Goñi \& Zulaika, 2000; Infante \& Goñi, 2009; Infante \& Zulaika, 2008; Moreno, Moreno \& Cervelló, 2007; Murgui, García \& García, 2016; Pezzuti, Pirouz \& Pechmann, 2015; Rodríguez-García, Gálvez, García-Cantó, PérezSoto, Rosa, Tárraga...Tárraga, 2015; Rodríguez-García, Tárraga, Rosa, García-Cantó, Pérez-Soto, Gálvez \& Tárraga, 2014; Rosa, García \& Carrillo 2019; SánchezAlcaraz \& Gómez-Mármol, 2014). El carácter multidimensional explica que sea un aspecto clave en el desarrollo del individuo no sólo a nivel físico, sino también emocional, familiar, académico o social (Axpe, Infante \& Goñi, 2016). A mayor nivel de práctica de actividad física el autoconcepto físico del alumnado aumenta en sus diferentes escalas (Álvarez, Cuevas, Lara \& González, 2015; Dieppa, Machargo, Luján \& Guillén 2008; Infante \& Goñi, 2009; Liu, Wu \& Ming, 2015; Menéndez \& González, 2019; Rosa, 2015).

La relación entre el autoconcepto físico y la actividad físico-deportiva tiene un carácter bidireccional: las autopercepciones físicas determinan la frecuencia, la duración y la intensidad de la actividad físico-deportiva y, al mismo tiempo, ésta incide en el autoconcepto físico (Chanal, Marsh, Sarrazin \& Bois, 2005; Marsh \& Craven, 2006; Marsh \& Perry, 2005; Trautwein, Gerlach \& Lüdtke, 2008). Por tanto, una actividad física adecuadamente planificada podría mejorar el autoconcepto.

Debido a la importancia que tiene el autoconcepto físico entre el alumnado de Educación Primaria (EP) y Educación Secundaria Obligatoria (ESO) y dada la influencia que tiene este con la práctica de actividad física escolar y extraescolar, se plantearon como objetivos de esta investigación analizar el autoconcepto físico del alumnado de sexto de EP y primero de ESO en función de i) las características sociodemográficas (edad y sexo), ii) del contexto escolar (tipo de centro, oferta educativa del centro y curso escolar), iii) y del nivel de práctica actividad físico-deportiva que realizan semanalmente los escolares.

\section{Método}

Se realizó una investigación con una estrategia asociativa y con un diseño de cohorte transversal (Ato, López \& Benavente, 2013), donde la toma de datos se realizó mediante una encuesta.

\section{Participantes}

La población objeto de estudio ha sido de 3227 estudiantes, 1527 escolares de sexto de Educación Primaria y 1700 escolares de primero de Educación Secundaria Obligatoria. El muestreo utilizado ha sido causal o incidental en el que han participado 1093 estudiantes, 620 de EP y 473 de ESO, de 19 centros educativos públicos y concertados de la ciudad de Badajoz. con un error del 2,4\% al 95\% de confianza.

\section{Variables}

En este estudio se tuvieron en cuenta variables sociodemográficas y contextuales como: edad, sexo, curso académico y tipo y carácter del centro educativo. 


\section{Instrumentos}

Para este estudio se diseñó un instrumento con un apartado de preguntas sociodemográficas creado ad hoc con dos cuestionarios:

a) Cuestionario de Autoconcepto Físico (CAF) creado por Goñi, Ruiz de Azúa \& Liberal (2004). El CAF es un cuestionario que consta de 36 ítems distribuidos en cuatro escalas específicas de autoconcepto físico (Habilidad Física, Condición Física, Atractivo Físico y Fuerza) y dos escalas generales (Autoconcepto Físico General y Autoconcepto General). La fiabilidad de las escalas de este cuestionario en la muestra han sido las siguientes: en Habilidad Física un $\alpha=.85$, en Condición física un $\alpha=.88$, en Atractivo Físico un $\alpha=.87$, en Fuerza un $\alpha=.84$, en Autoconcepto Físico General un $\alpha=.86$. y en Autoconcepto General un $\alpha=.84$. La fiabilidad del cuestionario CAF para este estudio, medida a través del coeficiente de Alpha de Crombach, es buena, $\alpha=.89$; aunque ligeramente inferior a la obtenida en el estudio de Goñi, Ruiz de Azua y Rodríguez (2006), $\alpha=.95$.

b) Cuestionario PAQ-C (Physical Activity Questionnaire for Children) propuesto por Kowalski, Crocker \& Faulkner (1997) para valorar la actividad físico deportiva que los escolares realizaron en los últimos 7 días durante su tiempo libre, las clases de educación física, así como en diferentes horarios durante los días de clase (comida, tardes y noches) y durante el fin de semana. El cuestionario está formado por nueve preguntas que valoran distintos aspectos de la actividad físico deportiva realizada por el alumnado mediante una escala tipo Likert de 5 puntos. La fiabilidad obtenida en el estudio ha sido de $\alpha=.81$ superior a la obtenida por Martínez-Gómez, \& col. (2009).

Posteriormente, a partir de los puntos de corte establecidos para el cuestionario PAQ-C por Benítez-Porres, López, Barrera-Expósito, Alvero-Cruz, Carnero, (2015), los estudiantes fueron clasificados en sedentarios (puntuación < 1.25), irregularmente activos (puntuación $\geq 1.25 y \leq 4.01$ ) y activos (puntuación > 4.01).

\section{Procedimiento}

En primer lugar, se contactó con el equipo directivo de cada uno de los centros educativos tanto públicos como concertados de la ciudad de Badajoz para explicarles el objetivo de la investigación. Tras su consentimiento, se les remitió una carta a los padres/madres/ tutores de los estudiantes solicitándoles su consentimiento para que su hijo/a participara en el estudio. A continuación, se procedió a aplicar los cuestionarios en los centros educativos con la colaboración de 6 eva- luadores externos debidamente entrenados. Se incidió en que contestaran con la mayor sinceridad posible, informándoles de que sus datos serían tratados de forma totalmente confidencial. La duración aproximada para cumplimentar el cuestionario fue de 15 minutos.

\section{Análisis estadístico}

A través de la prueba de Kolmogorov Smirnov se comprobó que las variables objeto de estudio no cumplían el supuesto de normalidad, tomando la decisión de realizar pruebas no paramétricas. A continuación, se realizó un estudio correlacional a través del coeficiente de correlación de Spearman de los factores de las escalas CAF.

Seguidamente se buscaron diferencias significativas en las variables relacionadas con el autoconcepto físico a través de la prueba $U$ de Mann Whitney para las variables sexo, tipo de centro y curso, y a través de la prueba $H$ de Kruskal-Wallis para los grupos de edad y el tipo de oferta educativa; se exploraron las comparaciones entre grupos a través de la prueba $U$ de Mann Whitney empleando la corrección de Bonferroni (significativo $p<.017$ para la oferta de niveles y $p<.008$ para los grupos de edad). Los análisis se realizaron con el paquete estadístico SPSS versión 23. Se calculó el tamaño del efecto a través de la $d$ de Cohen (Lenhard \& Lenhard, 2016), interpretándolo a través de los rangos establecidos por Cohen (1988): < .000 (adverso), .000 - .199 (ningún efecto), .200 - .499 (pequeño), .500 - .799 (intermedio) y $.800-\geq 1.000$ (alto).

\section{Resultados}

Las correlaciones de Spearman obtenidas en el cuestionario CAF de la tabla 1 , muestran que los estudiantes que tienen puntuaciones más altas de condición física tienen una habilidad física más alta ( $r=.721 ; p$ $=.00$ ). Los estudiantes que tienen puntuaciones más altas de fuerza tienen unas puntuaciones más altas de habilidad física $(r=.621 ; p=.00)$ y de condición física $(r=.595 ; p=.00)$. Igualmente, los estudiantes que tienen puntuaciones más altas de AFG tienen puntuaciones más elevadas de habilidad física $(r=.600 ; p=.00)$, de condición física $(r=.584 ; p=.00)$ y fuerza $(r=.474$; $p=.00$ ). Por otro lado, los estudiantes que tienen puntuaciones más altas de AG tienen puntuaciones más elevadas de habilidad física $(r=.498 ; p=.00)$, de condición física $(r=.591 ; p=.00)$, fuerza $(r=.459 ; p=.00)$ y AFG $(r=.557 ; p=.00)$. En lo que respecta al atractivo físico, mientras las puntuaciones sean más altas, las puntuaciones de habilidad física $(r=.574 ; p=.00)$, de 
Tabla 1. Correlaciones de Spearman de los factores del autoconcepto.

\begin{tabular}{|c|c|c|c|c|c|c|c|}
\hline & & Habilidad física & Condición física & Fuerza & AFG & AG & Atractivo física \\
\hline \multirow{2}{*}{ Habilidad física } & $r$ & 1.000 & & & & & \\
\hline & $p$ & & & & & & \\
\hline \multirow{2}{*}{ Condición física } & $r$ & $.721 * *$ & 1.000 & & & & \\
\hline & $p$ & .000 & & & & & \\
\hline \multirow{2}{*}{ Fuerza } & $r$ & $.621 * *$ & $.595^{* *}$ & 1.000 & & & \\
\hline & $p$ & .000 & .000 & & & & \\
\hline \multirow{2}{*}{ AFG } & $r$ & $.600 * *$ & $.584 * *$ & $.474 * *$ & 1000 & & \\
\hline & $p$ & .000 & .000 & .000 & & & \\
\hline \multirow{2}{*}{ AG } & $r$ & $.498^{* *}$ & $.591 * *$ & $.459 * *$ & $.557 * *$ & 1.000 & \\
\hline & $p$ & .000 & .000 & .000 & .000 & . & \\
\hline \multirow{2}{*}{ Atractivo físico } & $r$ & $.574 * *$ & $.594 * *$ & .466 ** & $.699 * *$ & $.564 * *$ & 1.000 \\
\hline & $p$ & .000 & .000 & .000 & .000 & .000 & . \\
\hline
\end{tabular}

Tabla 2. Correlaciones de las formas del autoconcepto físico controladas por el sexo y edad.

\begin{tabular}{|c|c|c|c|c|c|c|c|c|}
\hline \multicolumn{2}{|c|}{ Variables de control } & & Habilidad física & Condición física & Fuerza & AFG & AG & Atractivo físico \\
\hline \multirow{12}{*}{$\begin{array}{c}\text { Edad } \\
\text { y sexo }\end{array}$} & \multirow{2}{*}{ Habilidad física } & $r$ & 1.000 & & & & & \\
\hline & & $p$ & & & & & & \\
\hline & \multirow{2}{*}{ Condición física } & $r$ & $.765 * *$ & 1.000 & & & & \\
\hline & & $p$ & .000 & & & & & \\
\hline & \multirow{2}{*}{ Fuerza } & $r$ & $.638 * *$ & $.607 * *$ & 1.000 & & & \\
\hline & & $p$ & .000 & .000 & & & & \\
\hline & \multirow{2}{*}{ AFG } & $r$ & $.651 * *$ & $.657 * *$ & $.485 * *$ & 1.000 & & \\
\hline & & $p$ & .000 & .000 & .000 & & & \\
\hline & \multirow{2}{*}{ AG } & $r$ & $.570 * *$ & $.628 * *$ & $.459 * *$ & $659 * *$ & 1.000 & \\
\hline & & $p$ & .000 & .000 & .000 & 000 & & \\
\hline & \multirow{2}{*}{ Atractivo físico } & $r$ & $.598 * *$ & $.628 * *$ & $.490 * *$ & $785 * *$ & $.635 * *$ & 1.000 \\
\hline & & $p$ & .000 & .000 & .000 & .000 & .000 & . \\
\hline
\end{tabular}

condición física $(r=.594 ; p=.00)$, fuerza $(r=.466 ; p=$ $.00)$, AFG $(r=.699 ; p=.00)$ y Atractivo físico $(r=.564$ $p=.00)$ más altas serán.

En las correlaciones del autoconcepto físico en función del sexo y edad de la tabla 2 se puede observar cómo los estudiantes que tienen puntuaciones altas de alguna escala del autoconcepto físico obtienen puntuaciones elevadas del resto de escalas.

A continuación, se muestran los resultados del análisis descriptivo e inferencias de cada una de las escalas del autoconcepto físico en función de las variables sociodemográficas analizadas.

En la tabla 3 se presentan los resultados del análisis descriptivo e inferencial de la variable Atractivo físico. Se observan diferencias significativas en la variable atractivo físico en función de la edad, de la oferta de niveles educativos del centro y del curso académico ( $p=$ .00). Obtienen mayor puntuación de atractivo los chicos/as que tienen 13 años con respecto a los de 11 ( $p=$ $.00 ; d=.189$ ) y los de 12 años con respecto a los de 11 ( $p$ $=.002 ; d=.232$ ); los chicos/as que se encuentran matriculados en centros donde solo hay ESO en comparación con los centros donde solo se imparte Ed. Primaria ( $p=$ $.00 ; d=.246)$, y los escolares de $1^{\circ}$ de ESO en comparación con los de $6^{\circ}$ Ed. Primaria $(p=.00 ; d=.174)$.
En la tabla 4 se presentan los resultados del análisis descriptivo e inferencial de la variable habilidad física. Se observan diferencias significativas en la variable habilidad física en función del sexo, de la edad, del carácter del centro educativo, de la oferta de niveles educativos del centro y del curso académico. Obtienen mayor puntuación de habilidad física los chicos con respecto a las chicas ( $p=.00 ; d=.216)$, los que tienen 12 años con respecto a los de 11 ( $p=.004 ; d=.301)$; los que se encuentran matriculados en centros donde solo hay ESO en comparación con los centros donde solo se imparte Ed. Primaria o en centros donde hay ambas opciones ( $p=.00 ; d=.263)$; los que se encuentran matriculados en centros concertados en relación con los que se encuentran matriculados en centros públicos ( $p=.048 ; d$ $=.106)$ y los que están matriculados en $1^{\circ}$ de ESO con respecto a $6^{\circ}$ de Ed. Primaria $(p=.007 ; d=.201)$.

En la tabla 5 se presentan los resultados del análisis descriptivo e inferencial de la variable condición física. Se observan diferencias significativas en la variable condición física en función del sexo, de la edad, de la oferta de niveles educativos del centro y del curso académico $(p<.05)$. Obtienen mayor puntuación de condición física los estudiantes en comparación de las alumnas ( $p=.00 ; d=.275)$; los que tienen 12 años con 
Tabla 3. Análisis descriptivo e inferencias de la escala atractivo físico.

\begin{tabular}{|c|c|c|c|c|c|c|c|c|c|}
\hline & $\mathbf{n}$ & M & Rango Prom. & U & p & $\mathbf{H}$ & gl & p & d \\
\hline Sexo & & & & 137373 & .707 & & & & .139 \\
\hline Hombre & 550 & 4.05 & 550.55 & & & & & & \\
\hline Mujer & 543 & 404 & 543.41 & & & & & & \\
\hline Edad & & & & & & 29.856 & 3 & $.000 * *$ & .318 \\
\hline 11 & 434 & 4.1 & 600.89 & & & & & & \\
\hline 12 & 464 & 3.98 & 523.45 & & & & & & \\
\hline 13 & 164 & 3.81 & 558.61 & & & & & & \\
\hline 14 & 30 & 4.20 & 607.87 & & & & & & \\
\hline Tipo de Centro & & & & 147032.5 & .132 & & & & .091 \\
\hline Público & 687 & 4.00 & 535.98 & & & & & & \\
\hline Concertado & 406 & 4.11 & 565.56 & & & & & & \\
\hline Oferta de niveles & & & & & & 34.030 & 2 & $.000 * *$ & .348 \\
\hline Solo Primaria & 391 & 4.20 & 595.69 & & & & & & \\
\hline Solo ESO & 294 & 3.76 & 458.23 & & & & & & \\
\hline Ambas opciones & 408 & 4.11 & 564.31 & & & & & & \\
\hline Curso & & & & 122874.50 & $.000 * *$ & & & & .281 \\
\hline $6^{\circ}$ Primaria & 620 & 4.16 & 585.32 & & & & & & \\
\hline $1^{\circ} \mathrm{ESO}$ & 473 & 3.90 & 496.78 & & & & & & \\
\hline
\end{tabular}

Tabla 4. Análisis descriptivo e inferencias de la escala habilidad física.

\begin{tabular}{|c|c|c|c|c|c|c|c|c|c|}
\hline & $\mathbf{n}$ & M & Rango Prom. & U & p & H & gl & p & d \\
\hline Sexo & & & & 125090.50 & $.000 * *$ & & & & .284 \\
\hline Hombre & 550 & 4.12 & 591.06 & & & & & & \\
\hline Mujer & 543 & 3.88 & 502.37 & & & & & & \\
\hline Edad & & & & & & 16.665 & 3 & $.002 * *$ & .225 \\
\hline 11 & 434 & 4.12 & 591.13 & & & & & & \\
\hline 12 & 464 & 3.91 & 516.35 & & & & & & \\
\hline 13 & 164 & 3.91 & 511.91 & & & & & & \\
\hline 14 & 30 & 4.06 & 560.98 & & & & & & \\
\hline Tipo de Centro & & & & 149421.5 & $.048 * *$ & & & & .12 \\
\hline Público & 687 & 3.95 & 532.50 & & & & & & \\
\hline Concertado & 406 & 4.10 & 571.53 & & & & & & \\
\hline Oferta de niveles & & & & & & 21.002 & 2 & $.000 * *$ & .266 \\
\hline Solo Primaria & 391 & 4.05 & 576.25 & & & & & & \\
\hline Solo ESO & 294 & 3.81 & 475.19 & & & & & & \\
\hline Ambas opciones & 408 & 4.09 & 570.72 & & & & & & \\
\hline Curso & & & & 132669.50 & $.007 * *$ & & & & .164 \\
\hline $6^{\circ}$ Primaria & 620 & 4.06 & 569.47 & & & & & & \\
\hline $1^{\circ} \mathrm{ESO}$ & 473 & 3.93 & 517.55 & & & & & & \\
\hline
\end{tabular}

Tabla 5. Análisis descriptivo e inferencias de la escala condición física.

\begin{tabular}{|c|c|c|c|c|c|c|c|c|c|}
\hline & $\mathbf{n}$ & M & Rango Prom. & $\mathbf{U}$ & $p$ & H & gl & $\mathbf{p}$ & d \\
\hline Sexo & & & & 122527 & $.000 * *$ & & & & .315 \\
\hline Hombre & 550 & 4.13 & 595.72 & & & & & & \\
\hline Mujer & 543 & 3.85 & 497.65 & & & & & & \\
\hline Edad & & & & & & 29.684 & 3 & $.000 * *$ & .317 \\
\hline 11 & 434 & 4.13 & 596.03 & & & & & & \\
\hline 12 & 464 & 3.86 & 496.45 & & & & & & \\
\hline 13 & 164 & 3.95 & 533.18 & & & & & & \\
\hline 14 & 30 & 4.31 & 583.82 & & & & & & \\
\hline Tipo de Centro & & & & 144724 & .295 & & & & .063 \\
\hline Público & 687 & 3.95 & 539.34 & & & & & & \\
\hline Concertado & 406 & 4.06 & 559.96 & & & & & & \\
\hline Oferta de niveles & & & & & & 11.779 & 2 & $.003 * *$ & .19 \\
\hline Solo Primaria & 391 & 4.07 & 573.81 & & & & & & \\
\hline Solo ESO & 294 & 3.80 & 494.12 & & & & & & \\
\hline Ambas opciones & 408 & 4.06 & 559.40 & & & & & & \\
\hline Curso & & & & 136420 & $.048^{* *}$ & & & & .12 \\
\hline $6^{\circ}$ Primaria & 620 & 4.05 & 563.47 & & & & & & \\
\hline $1^{\circ} \mathrm{ESO}$ & 473 & 3.92 & 525.41 & & & & & & \\
\hline
\end{tabular}


Tabla 6. Análisis descriptivo e inferencias de la escala fuerza.

\begin{tabular}{|c|c|c|c|c|c|c|c|c|c|}
\hline & $\mathbf{n}$ & M & Rango Prom. & $\mathbf{U}$ & p & $\mathbf{H}$ & gl & $\mathbf{p}$ & d \\
\hline Sexo & & & & 128631 & $.000 * *$ & & & & .242 \\
\hline Hombre & 550 & 3.84 & 584.63 & & & & & & \\
\hline Mujer & 543 & 3.65 & 508.89 & & & & & & \\
\hline Edad & & & & & & 28.071 & 3 & $.000 * *$ & .307 \\
\hline 11 & 434 & 3.84 & 579.27 & & & & & & \\
\hline 12 & 464 & 3.63 & 502.94 & & & & & & \\
\hline 13 & 164 & 3.73 & 546.48 & & & & & & \\
\hline 14 & 30 & 4.22 & 747.88 & & & & & & \\
\hline Tipo de Centro & & & & 138087.5 & .785 & & & & .016 \\
\hline Público & 687 & 3.74 & 549.00 & & & & & & \\
\hline Concertado & 406 & 3.76 & 543.62 & & & & & & \\
\hline Oferta de niveles & & & & & & 14.310 & 2 & $.001 * *$ & .214 \\
\hline Solo Primaria & 391 & 3.84 & 588.48 & & & & & & \\
\hline Solo ESO & 294 & 3.60 & 496.78 & & & & & & \\
\hline Ambas opciones & 408 & 3.76 & 543.43 & & & & & & \\
\hline Curso & & & & 133715.50 & $.012 * *$ & & & & .152 \\
\hline $6^{\circ}$ Primaria & 620 & 3.80 & 567.83 & & & & & & \\
\hline $1^{\circ} \mathrm{ESO}$ & 473 & 3.67 & 519.70 & & & & & & \\
\hline
\end{tabular}

Tabla 7. Análisis descriptivo e inferencias del autoconcepto físico general.

\begin{tabular}{|c|c|c|c|c|c|c|c|c|c|}
\hline & $\mathrm{n}$ & $\mathrm{M}$ & Rango Prom. & U & $\mathrm{p}$ & $\mathrm{H}$ & gl & $\mathrm{p}$ & d \\
\hline Sexo & & & & 141797 & .147 & & & & .087 \\
\hline Hombre & 550 & 4.12 & 560.69 & & & & & & \\
\hline Mujer & 543 & 4.07 & 533.14 & & & & & & \\
\hline Edad & & & & & & 18.854 & 3 & $.001 * *$ & .243 \\
\hline 11 & 434 & 4.21 & 597.01 & & & & & & \\
\hline 12 & 464 & 4.03 & 516.44 & & & & & & \\
\hline 13 & 164 & 3.98 & 504.03 & & & & & & \\
\hline 14 & 30 & 4.06 & 525.53 & & & & & & \\
\hline Tipo de Centro & & & & 140275.5 & .874 & & & & .01 \\
\hline Público & 687 & 4.07 & 545.84 & & & & & & \\
\hline Concertado & 406 & 4.14 & 548.96 & & & & & & \\
\hline Oferta de niveles & & & & & & 27.073 & 2 & $.000 * *$ & .307 \\
\hline Solo Primaria & 391 & 4.21 & 600.91 & & & & & & \\
\hline Solo ESO & 294 & 3.90 & 474.64 & & & & & & \\
\hline Ambas opciones & 408 & 4.13 & 547.99 & & & & & & \\
\hline Curso & & & & 123571.50 & $.000 * *$ & & & & .272 \\
\hline $6^{\circ}$ Primaria & 620 & 4.20 & 584.19 & & & & & & \\
\hline $1^{\circ} \mathrm{ESO}$ & 473 & 3.98 & 498.25 & & & & & & \\
\hline
\end{tabular}

respecto a los de 11 años $(p=.00 ; d=.361)$. También obtienen mayor puntuación los chicos/as que se encuentran matriculados en centros donde solo hay ESO en comparación con los centros donde solo se imparte Ed. Primaria ( $p=.003 ; d=.374$ ) o en centros donde hay ambas opciones ( $p=.020 ; d=.186)$; y los que están matriculados en $1^{\circ}$ de ESO con respecto a $6^{\circ}$ de Ed. Primaria $(p=.048)$.

En la tabla 6 se presentan los resultados del análisis descriptivo e inferencial de la variable fuerza. Se observan diferencias significativas en la variable fuerza en función del sexo, de la edad, de la oferta de niveles educativos del centro y del curso académico $(p<.05)$. Obtienen mayor puntuación de fuerza los estudiantes que las alumnas ( $p=.00 ; d=.305)$; los que tienen 12 años con respecto a los de 11 años ( $p=.003 ; d=.347$ ), los de 12 años con respecto a los de 14 años ( $p=.00 ; d$
$=.292)$ y los de 13 años con respecto a los de 14 años ( $p=.013 ; d=.187)$; los chicos/as que se encuentran matriculados en centros donde solo hay ESO en comparación con los centros donde solo se imparte Ed. Primaria ( $p=.00 ; d=.364)$; y los que están matriculados en $1^{\circ}$ de ESO con respecto a $6^{\circ}$ de Ed. Primaria ( $p=$ $.012 ; d=.241)$.

En la tabla 7 se presentan los resultados del análisis descriptivo e inferencial de la variable AFG. Se observan diferencias significativas en la variable $A F G$ en función de la edad, de la oferta de niveles educativos del centro y del curso académico $(p<.01)$. Obtienen mayor puntuación de AFG los chicos/as que tienen 12 años con respecto a los de 11 ( $p=.001 ; d=.358)$ y los de 13 años con respecto a los de 11 años ( $p=.013$; $d$ $=.297$ ); los que están matriculados en centros donde solo hay ESO en comparación con los centros donde 
Tabla 8. Análisis descriptivo e inferencias del autoconcepto general.

\begin{tabular}{|c|c|c|c|c|c|c|c|c|c|}
\hline & $\mathbf{n}$ & M & Rango Prom. & U & p & $\mathbf{H}$ & gl & p & d \\
\hline Sexo & & & & 148109.50 & .814 & & & & .014 \\
\hline Hombre & 550 & 4.33 & 549.21 & & & & & & \\
\hline Mujer & 543 & 4.31 & 544.76 & & & & & & \\
\hline Edad & & & & & & 14.302 & 3 & $.006 * *$ & .205 \\
\hline 11 & 434 & 4.41 & 590.74 & & & & & & \\
\hline 12 & 464 & 4.26 & 516.06 & & & & & & \\
\hline 13 & 164 & 4.26 & 518.96 & & & & & & \\
\hline 14 & 30 & 4.30 & 546.25 & & & & & & \\
\hline Tipo de Centro & & & & 149191 & .052 & & & & \\
\hline Público & 687 & 4.28 & 532.84 & & & & & & \\
\hline Concertado & 406 & 4.39 & 570.97 & & & & & & \\
\hline Oferta de niveles & & & & & & 20.288 & 2 & $.000 * *$ & .261 \\
\hline Solo Primaria & 391 & 4.38 & 575.14 & & & & & & \\
\hline Solo ESO & 294 & 4.15 & 476.71 & & & & & & \\
\hline Ambas opciones & 408 & 4.39 & 570.69 & & & & & & \\
\hline Curso & & & & 136746.50 & .054 & & & & .116 \\
\hline $6^{\circ}$ Primaria & 620 & 4.36 & 562.94 & & & & & & \\
\hline $1^{\circ} \mathrm{ESO}$ & 473 & 4.27 & 526.10 & & & & & & \\
\hline
\end{tabular}

Tabla 9: Asociación entre la actividad física y el autoconcepto físico.

\begin{tabular}{|c|c|c|c|c|c|c|c|c|}
\hline & & $\mathrm{n}$ & M & Rango Prom. & $\mathrm{H}$ & $\mathrm{gl}$ & $p$ & $d$ \\
\hline \multirow{4}{*}{ Habilidad física } & PAQ-C & & & & 11.138 & 2 & .004 & .184 \\
\hline & Sedentarios & 166 & 4.02 & 561.02 & & & & \\
\hline & Irregularmente activos & 890 & 3.98 & 537.58 & & & & \\
\hline & Activos & 37 & 4.39 & 710.70 & & & & \\
\hline \multirow{4}{*}{ Condición física } & PAQ-C & & & & 12.597 & 2 & .002 & .198 \\
\hline & Sedentarios & 166 & 3.87 & 511.19 & & & & \\
\hline & Irregularmente activos & 890 & 4.00 & 546.73 & & & & \\
\hline & Activos & 37 & 4.43 & 714.16 & & & & \\
\hline \multirow{4}{*}{ Fuerza } & PAQ-C & & & & 5.448 & 2 & .066 & .113 \\
\hline & Sedentarios & 166 & 3.79 & 561.02 & & & & \\
\hline & Irregularmente activos & 890 & 3.72 & 539.75 & & & & \\
\hline & Activos & 37 & 4.05 & 658.62 & & & & \\
\hline \multirow{4}{*}{ AFG } & PAQ-C & & & & 3.858 & 2 & .145 & .083 \\
\hline & Sedentarios & 166 & 4.06 & 525.89 & & & & \\
\hline & Irregularmente activos & 890 & 4.09 & 547.15 & & & & \\
\hline & Activos & 37 & 4.33 & 638.14 & & & & \\
\hline \multirow{4}{*}{ AG } & PAQ-C & & & & 13.613 & 2 & .001 & .208 \\
\hline & Sedentarios & 166 & 4.25 & 478.14 & & & & \\
\hline & Irregularmente activos & 890 & 4.33 & 555.05 & & & & \\
\hline & Activos & 37 & 4.50 & 662.24 & & & & \\
\hline \multirow{4}{*}{ Atractivo físico } & PAQ-C & & & & 4.921 & 2 & .085 & .104 \\
\hline & Sedentarios & 166 & 4.00 & 511.66 & & & & \\
\hline & Irregularmente activos & 890 & 4.04 & 550.03 & & & & \\
\hline & Activos & 37 & 4.34 & 632.70 & & & & \\
\hline
\end{tabular}

solo se imparte Ed. Primaria ( $p=.00 ; d=.212$ ) o en centros donde hay ambas opciones ( $p=.007 ; d=.289)$; y los que están matriculados en $1^{\circ}$ de ESO con respecto a $6^{\circ}$ de Ed. Primaria $(p=.00 ; d=.376)$.

En la tabla 8 se presentan los resultados del análisis descriptivo e inferencial de la variable Autoconcepto General (AG). Se observan diferencias significativas en la variable AG en función de la edad y la oferta de niveles educativos del centro $(p<.01)$. Obtienen mayor puntuación de AG los chicos/as que tienen 12 años con respecto a los de 11 ( $p=.004 ; d=.384)$. También obtienen mayor puntuación los chicos/as que se en- cuentran matriculados en centros donde solo hay ESO en comparación con los centros donde solo se imparte Ed. Primaria y en comparación con los centros donde hay ambas opciones ( $p=.00 ; d=.204)$.

A continuación, se muestra la asociación entre la actividad física y el autoconcepto físico. En la tabla 9 se muestran diferencias significativas de la actividad física con la escala de habilidad física $\left(X^{2}=11.138 ; \mathrm{gl}\right.$ $=2 ; p=.004)$, con la condición física $\left(X^{2}=12.597 ; g l\right.$ $=2 ; p=.002)$ y con la escala de actividad general $\left(X^{2}\right.$ $=13.613 ; g l=2 ; p=.001)$; En las comparaciones entre grupo realizadas los estudiantes activos obtienen me- 
jores puntuaciones que los irregularmente activos (HF $p=.003, d=.407$; CF $p=.005, d=.376$; y AG $p=.011, d$ $=.275)$ y que los sedentarios ( $\mathrm{HF} p=.027, d=.213 ; \mathrm{CF}$ $p=.001, d=.389 ;$ y AG $p=.004 ; d=.356)$.

\section{Discusión}

Un adecuado desarrollo del autoconcepto, en general, es importante para el bienestar de las personas (Penado \& Rodicio-García, 2017). Para conocer cómo se desarrolla el autoconcepto en la edad se plantearon como objetivos de esta investigación analizar este en función de i) las características sociodemográficas (edad y sexo), ii) del contexto escolar (tipo de centro, oferta educativa del centro y curso escolar), iii) y del nivel de práctica actividad físico-deportiva que realizan semanalmente los escolares.

Al analizar las características sociodemográficas, sexo y edad, objetivo número uno, se ha podido constatar que el Autoconcepto Físico es superior en los chicos que en las chicas, excepto en las escalas de atractivo físico, actividad física general y autoconcepto general. Sin embargo, en otros estudios los chicos tienen unas puntuaciones superiores a las de las chicas en todas las escalas del cuestionario de Autoconcepto físico (Infante \& Fernández-Zabala, 2015; Jacobs, Lanza, Osgood, Eccles \& Wigfield, 2002; Klomsten, Marsh \& Skaalvik 2005; Klomsten, Skaalvik \& Espens, 2004; Marsh, Gerlach, Trautwein, Ludtke \& Brettschneider, 2007; Moreno, Cervelló \& Moreno, 2008; Sánchez-Alcaraz \& Gómez-Marmol, 2014; Videra \& Reigal, 2003). En el estudio de Fraile y Catalina (2013) los chicos se perciben mucho más hábiles deportivamente, con mucha mejor condición física y con mucha más fuerza que las niñas.

Por otro lado, es necesario indicar que en otros estudios no encontraron diferencias significativas en función del sexo de las diferentes escalas del cuestionario (Guillen \& Ramírez, 2011; Molero, Ortega, Valiente \& Zagalaz, 2010).

En cuanto al atractivo físico, en este estudio no se observan diferencias en función del sexo. Es decir, tanto los chicos como las chicas están preocupados por igual por el atractivo físico. Sin embargo, en otros estudios los chicos obtenían puntuaciones más altas de atractivo físico que las chicas (Goñi et al., 2004; Lubans, Aguiar \& Callister, 2010; Molero, Zagalaz-Sánchez \& Cachón-Zagalaz, 2013; Videra \& Reigal (2003).

También se observa diferencias significativas en el autoconcepto en función de la edad, aunque con tamaño del efecto bajo al comparar todos los grupos. A medida que el escolar tiene más edad obtiene puntuaciones más elevadas en las diferentes escalas del auto- concepto. Resultados totalmente opuestos a los obtenidos por Pastor, Balaguer \& García-Merita (2003) ya que en dicho estudio a medida que aumenta la edad del alumnado empeora las puntuaciones de las diferentes escalas. Sin embargo, en los estudios de Dieppa, Machargo, Luján \& Guillén (2008) y Goñi et al. (2004) no se observan diferencias significativas del autoconcepto en función de la edad del alumnado.

En lo que respecta al segundo objetivo, no se han encontrado diferencias en el autoconcepto en función del tipo de centro, pero si teniendo en cuenta la oferta educativa del centro, es decir si el centro tiene diversas etapas educativas o sólo una, y teniendo en cuenta el curso escolar. En un estudio realizado en México por Salum-Fares, Reséndiz \& Saldivar (2012) se pudo constatar que el autoconcepto es más elevado en los centros privados que en los centros públicos.

Por otra parte, y respondiendo al objetivo número tres, se ha constatado que los estudiantes que realizan más actividad física obtienen mejores puntuaciones en algunas de las escalas del autoconcepto físico en la misma línea que ya apuntaron otros estudios (González \& Alvariñas, 2004; Pastor \& Balaguer; 2001). Este patrón de variación es igual en el caso de la relación entre el autoconcepto físico y la práctica físico-deportiva fuera del ámbito escolar, tal y como han demostrado estudios previos (Fox, 2000; Taylor \& Fox, 2005; Infante \& Zulaika, 2008).

En este estudio se confirma que la práctica regular de la actividad física mejora solo algunas dimensiones del autoconcepto físico (habilidad física, condición física y autoconcepto general), a diferencia de estudios anteriores realizados por Esnaola \& Zulaica (2009); Fox (2000); González \& Alvariñas (2004); Lindgren, Baigi, Apitzsch, \& Bergh (2011); Moreno, Cervelló \& Moreno (2008); Pastor \& Balaguer (2001); Ortega (2005); Piéron (2002). No se ha podido constatar que el nivel de práctica de actividad física haya mejorado las escalas de atractivo físico y fuerza, tal y como sucediera en Goñi \& Infante (2010) y Rodríguez \& Fernández (2005). En el caso de la fuerza, probablemente no baste con realizar actividad física, sino que sería necesario un programa específico para la mejora de esta variable.

Molero, Ortega, Valiente \& Zagalaz (2010) encontraron diferencias significativas en las escalas de Habilidad Física y Fuerza, coincidiendo solamente con nuestro estudio con la escala de habilidad física. Estos autores destacaron en su estudio una mayor valoración de la escala de fuerza por la práctica de actividad física mientras que en nuestro estudio destacamos las mejoras en la escala de condición física y AG. Por otro lado, Reigal, Videra, Parra \& Juárez (2012) observaron 
diferencias significativas entre la frecuencia de práctica de actividad física extraescolar y algunas escalas del autoconcepto físico (habilidad física, condición física, atractivo físico, fuerza y autoconcepto físico general) coincidiendo únicamente con nuestro estudio en las escalas de habilidad física y condición física.

\section{Aplicaciones prácticas}

Es necesario favorecer en la educación física escolar y en la actividad física extraescolar la mejora de los distintos compontes del autoconcepto físico. Para ello el docente deberá utilizar programas motivantes, lúdicos y ajustados a las necesidades de los escolares para la mejora de la condición física y la fuerza en el ámbito escolar. Proponer actividades físico deportivas donde los escolares puedan aplicar sus habilidades físicas sin miedo a ser juzgados, donde puedan adaptar sus capacidades para la resolución de las mismas. Además, será necesario crear un clima en el aula donde el trabajo escolar esté orientado hacia la tarea y no hacia el ego, motivando la participación y la mejora personal, valorando en la práctica las propias capacidades y las de los demás.

\section{Conclusión}

En este estudio se encontrado diferencias en algunas formas de autoconcepto en función del sexo, edad, curso educativo y oferta de los centros escolares. Los chicos obtienen puntuaciones más elevadas en la habilidad física, condición física y fuerza que las chicas. A medida que aumenta la edad de los escolares las puntuaciones de autoconcepto físico aumentan. Se obtienen mayores puntuaciones de autoconcepto en los centros donde solo se imparte ESO en comparación con los centros donde solo se imparte Ed. Primaria o en los centros donde se impartan ambas opciones (Primaria y Secundaria). Los estudiantes de $1^{\circ}$ de ESO obtienen puntuaciones superiores a las obtenidas por el alumnado de $6^{\mathrm{a}}$ de Ed. Primaria.

Los estudiantes activos físicamente obtienen mayores puntuaciones de habilidad física, condición física y autoconcepto general que los estudiantes irregularmente activos y los sedentarios.

\section{Limitaciones del estudio y prospectivas}

El presente estudio adolece de una serie de limitaciones que han de ser consideradas en futuros trabajos científicos entre las que destaca que la muestra de estudio solo estaba compuesta por escolares que cursaban $6^{\circ}$ de Educación Primaria y $1^{\circ}$ de Educación Secundaria Obligatoria, por lo que sería conveniente conocer la opinión de todos los estudiantes de Educación Primaria y Educación Secundaria Obligatoria de la ciudad de Badajoz, e incluso ampliar el muestreo a la Comunidad Autónoma de Extremadura. Con el tamaño muestral de los grupos comparados y los resultados obtenidos el tamaño del efecto obtenido es pequeño (valores entre 0.1-0.4), aunque estos resultados indican tendencias que deben ser contrastadas con futuros trabajos. También sería conveniente conocer el autoconcepto físico de los sujetos en un momento determinado y tras aplicar un programa de actividad física observar si las diferentes puntuaciones mejoran o no. 
Álvarez, L., Cuevas, R., Lara, A., \& González, J. (2015). Diferencias del autoconcepto físico en practicantes y no practicantes de actividad física en estudiantes universitarios. Cuadernos de Psicología del Deporte, 15(2), 27-34. https://doi.org/10.4321/s1578-84232015000200004

Alvariñas, M., \& González, M. (2004). Relación entre la práctica físicodeportiva extraescolar y el autoconcepto físico en la adolescencia. Revista de Educación Física, 94(1), 5-8.

Ato, M., López, J. J., \& Benavente, A. (2013). Un sistema de clasificación de los diseños de investigación en psicología. Anales de Psicología, 29(3), 1038-1059. https://doi.org/10.6018/analesps.29.3.178511

Axpe, I., Infante, G., \& Fernández-Zabala, A. (2015). ¿Es posible mejorar el autoconcepto físico universitario mediante una intervención cognitiva? Acción psicológica 12(1), 23-34. https://doi.org/10.5944/ ap.12.1.11864

Axpe, I., Infante, G., \& Goñi, E. (2016). Mejora del autoconcepto físico. Eficacia de una intervención cognitiva breve con alumnado universitaria de Educación Primaria. Educación XX1, 19(1), 227-246.

Barrios, D., Gómez, M.A., \& Barriopedro, M.I. (2017). Análisis del autoconcepto físico en estudiantes de enseñanza física que participan en el proyecto de especialización deportiva de la Comunidad de Madrid. Revista de Psicología del Deporte, 26(2), 45-53.

Benítez-Porres, J., López, I., Barrera-Expósito J., Alvero-Cruz, J.R., \& Carnero, E. A. (2015). Puntos de corte para clasificar adolescentes activos a través del cuestionario de actividad física para adolescentes (PAQ-A). Gymnasium, 1(1), 71-73.

Blanco, H., Benavides, E.V., Tristán, J.L., \& Mayorga-Vega, D. (2017). Actividad física, imagen corporal y autoconcepto personal en jóvenes universitarias mexicanas. Revista de Psicología del Deporte, 26, (Suppl 2), 25-33.

Burns, R. B. (1990). El autoconcepto. Teoría, medición, desarrollo y comportamiento. Bilbao: Ediciones EGA.

Candel, N., Olmedilla, A., \& Blas, A. (2008). Relaciones entre la práctica de actividad física y el autoconcepto, la ansiedad y la depresión en chicas adolescentes. Cuadernos de Psicología del Deporte, 8(1), 61-77.

Chanal, J. P., Marsh, H. W., Sarrazin, P. G., \& Bois, J. E. (2005). Big-fishlittlepond effects on gymnastics self-concept: Social comparison processes in a physical setting. Journal of Sport and Exercise Psychology, 27(1), 53-70. https://doi.org/10.1123/jsep.27.1.53

Cohen, J. (1988). Statistical power analysis for the behavioral sciences (2 Ed.). Hillsdale, NJ: Erlbaum.

Contreras, O. R., Fernández, J. G., García, L. M., Palou, P., \& Ponseti, J. (2010). El autoconcepto físico y su relación con la práctica deportiva en estudiantes adolescentes. Revista de Psicología del Deporte, 19, 23-39.

Dieppa, M., Machargo, J., Luján, I., \& Guillén, F. (2008). Autoconcepto general y físico en jóvenes españoles y brasileños que practican actividad física vs. no practicantes. Revista de Psicología del Deporte, 17(2), 221-239.

Esnaola, I. (2005). Desarrollo del autoconcepto durante la adolescencia y principio de la juventud. Revista de Psicología General y Aplicada, 58(2), 265-277

Esnaola, I., \& Zulaika, L. M. (2009). Physical activity and physical selfconcept in a sample of middle-aged Basque adults. Perceptual and Motor Skills, 108(2), 479-490. https://doi.org/10.2466/pms.108.2.479-490

Esnaola, I., Infante, G., \& Zulaika, L. (2011). The Multidimensional Structure of Physical Self-Concept. The Spanish Journal of Psychology, 14(1), 304-312. https://doi.org/10.5209/rev_sjop.2011.v14.n1.27

Esnaola, I., Rodríguez, A., \& Goñi, E. (2011). Propiedades psicométricas del cuestionario de Autoconcepto AF5. Anales de Psicología, 27(1), 109-117

Espejo, T., Zurita, F., Chacón, R., Castro, M., Martínez-Martínez, A., \& Pérez-Cortés, A.J. (2018). Actividad física y autoconcepto: dos factores de estudio en adolescentes de zona rural. Revista iberoamericana de psicología del ejercicio y el deporte, 13(2), 203-210.

Fox, K. (2000). The effects of exercise on self-perceptions and self-esteem. En Biddle, S.J.H., Fox, K. R. y Boutcher, S.H. (Eds.), Physical activity and Psychological wellbeing (pp. 88-117). London: Routledge and Kegan Paul.
Fox, K. R. (1990). The physical self-perception profile manual. Dekalb, IL: Northem Illionois University Office for health promotion.

Fraguela-Vale, R., Varela-Garrote, L., \& Sanz-Arazuri, E. (2016). Ocio deportivo, imagen corporal y satisfacción vital en jóvenes españoles. Revista de Psicología del Deporte, 25(2), 33-38.

Fraile, A., \& Catalina, J. (2013). Diferencias en autoconcepto físico en escolares de primaria y secundaria. Lúdica pedagógica, 1(18), 93-102. https://doi.org/10.17227/01214128.18ludica93.102

Gálvez, A., Rodríguez-García, P.L., Rosa, A., García-Cantó, E., PérezSoto, J.J., Tárraga, P.J., \& Tárraga, M.L. (2016). Capacidad aeróbica, estado de peso y autoconcepto en escolares de primaria. Clínica e Investigación en Arteriosclerosis, 28(1), 1-8.

Garaigordobil M., Pérez, J.I., \& Mozaz, M. (2008). Self-concept, self-esteem and psychopathological symptoms. Psicothema, 20(2), 114-123.

González, G., \& Fernández, F.J. (2017). Perspectiva cualitativa y cuantitativa del autoconcepto físico y la imagen corporal de los diferentes profesionales de la actividad física y del deporte. Revista de Psicología del Deporte, 26(2), 105-111.

González, M. A., \& Alvariñas, M. (2004). Relación entre la práctica físicodeportivo extraescolar y el autoconcepto físico en la adolescencia. Revista de Educación Física: renovar la teoría y práctica, 94(1), 5-8.

Goñi, A., \& Zulaika, L.M. (2000). Relationships between physical education clases and the enhancement of fifth grade pupils self- concept. Perceptual and motor and skills, 91(1), 146-150. https://doi. org/10.2466/pms.2000.91.1.246

Goñi, A., \& Infante, G. (2010). Actividad físico-deportiva, autoconcepto físico y satisfacción con la vida. European Journal o Education and Psychology, 3(2), 199-208. https://doi.org/10.30552/ejep.v3i2.52

Goñi, A., Ruiz de Azúa, S., \& Liberal, I. (2004). Propiedades psicométricas de un nuevo cuestionario para la medida del autoconcepto físico. Revista Psicología del Deporte vol. 13(2), 195-213.

Goñi, E., Esnaola, I., Rodríguez, A., \& Camino, I. (2015). Personal selfconcept and satisfaction with life in adolescence, youth and adulthood. Psicothema, 27(1), 52-58. doi: 10.7334/psicothema2014.105

Guillén, F., \& Ramírez, M. (2011). Relación entre el autoconcepto y la condición física en alumnos del Tercer Ciclo de Primaria. Revista de Psicología del deporte, 20(1), 45-59.

Infante, G., \& Goñi, A. (2009). Actividad físico-deportiva y autoconcepto físico en la edad adulta. Revista psicodidáctica, 14(1), 49-62.

Infante, G., \& Zulaika, L. (2008). Actividad física y autoconcepto físico. En A. Goñi (Coord.), El autoconcepto físico (pp. 125-153). Madrid: Pirámide.

Jacobs, J. E., Lanza, S., Osgood, D. W., Eccles, J. S., \& Wigfield, A. (2002) Changes in children's self-competence and values: Gender and domain differences across grades one through twelve. Child Development, 73(1), 509-527. https://doi.org/10.1111/1467-8624.00421

Klomsten, A. T., Skaalvik, E. M., \& Espens, G. A. (2004). Physical selfconcept and sports: do gender differences still exist? Sex Roles, 50(1), 119-127. https://doi.org/10.1023/b:sers.0000011077.10040.9a

Klomsten, A., Marsh, H., \& Skaalvik, M. (2005). Adolescents' perceptions of masculine and feminine values in sport and physical education: a study of gender differences. Sex Roles, 52(9), 625-636. https:// doi.org/10.1007/s11199-005-3730-x

Kowalski, K.C., Crocker, P.R., \& Faulkner, R.A. (1997). Validation sf the physical activity questionnaire for older children. Pediatric exercise science, 9(2), 174-186. https://doi.org/10.1123/pes.9.2.174

Lenhard, W., \& Lenhard, A. (2016). Calculation of Effect Sizes. Dettelbach: Psicometría. doi: 10.13140 / RG.2.1.3478.4245

Lindgren, E., Baigi, A., Apitzsch, E., \& Bergh, H. (2011). Impact of a sixmonth empowermentbased exercise intervention programme in nonphysically active adolescent Swedish girls. Health Education Journal, 70(1), 920.

Liu, M., Wu, L., \& Ming, Q. (2015). How Does Physical Activity Intervention Improve Self-Esteem and Self-Concept in Children and Adolescents? Evidence from a Meta-Analysis. Plos One, 10(8), e0134804.

Lubans, D. R., Aguiar, E. J., \& Callister, R. (2010). The effects of free weights and elastic tubing resistance training on physical self-percep- 
tion in adolescents. Psychology of Sport and Exercise, 11(1), 497-504. https://doi.org/10.1016/j.psychsport.2010.06.009

Marsh, H. W. (1998). Age and Gender effects in physical self- concept for adolescent elite athletes and Nonathletes. A multicohort - multioccasion design. Joumal of Sport and exercise Psychology, 20(2), 237-259.

Marsh, H. W., \& Craven, R. G. (2006). Reciprocal effects of self-concept and performance from a multidimensional perspective: Beyond seductive pleasure and unidimensional perspectives. Perspectives on Psychological Science, 1(2), 133-163. https://doi.org/10.1111/j.17456916.2006.00010.x

Marsh, H. W., \& Perry, C. (2005). Does a positive self-concept contribute to winning gold medals in elite swimming? The causal ordering of elite athlete self-concept and championship performances. Journal of Sport and Exercise Psychology, 27(1), 71-91. https://doi.org/10.1123/ jsep.27.1.71

Marsh, H. W., Gerlach, E., Trautwein, U., Ludtke, O., \& Brettschneider, W. (2007). Longitudinal study of preadolescent sport self-concept and performance: reciprocal and causal ordering. Child Development, 78(1), 1640-1656. https://doi.org/10.1111/j.1467-8624.2007.01094.x

Martinez-Gómez, D., Martinez-de-Haro, V., Pozo, T., Welk, G.J., Villagra, A., Calle, M. E., Marcos, A., \& Veiga, O.L. (2009). Fiabilidad y validez del cuestionario de actividad PAQ-A en adolescentes españoles. Revista Española de Salud Pública, 83(3), 427-439. https://doi.org/10.1590/ s1135-57272009000300008

Menéndez, D., \& González, C. (2019). Relaciones entre la práctica de actividad física y deportiva, el autoconcepto, la imagen corporal y los hábitos alimentarios en estudiantes de primaria. E-balonmano.com: Revista de Ciencias del Deporte, 15(1), 79-96. http://www.e-balonmano.com/ojs/index.php/revista/index

Molero, D., Ortega, F., Valiente, I., \& Zagalaz, M. L. (2010). Estudio comparativo del autoconcepto físico en adolescentes en función del género y del nivel de actividad físico-deportiva. Retos. Nuevas tendencias en Educación Física, Deporte y Recreación 17(1), 38-41.

Molero, D., Zagalaz-Sánchez, M. L., \& Cachón-Zagalaz, J. (2013). Estudio comparativo del autoconcepto físico a lo largo del ciclo vital. Revista de Psicología del Deporte, 22(1), 135-142.

Moreno, J. A., Cervelló, E., \& Moreno, R. (2008). Importancia de la práctica físico-deportiva y del sexo en el autoconcepto físico de los 9 a los 23 años. International Journal of Clinical and Health Psychology, 8(1), 171-183.

Moreno, J. A., Moreno, R., \& Cervelló, E. (2007). El autoconcepto físico como predictor de la intención de ser físicamente activo. Revista de Psicología y Salud, 17(2), 261-267.

Murgui, S., García, C., \& García, A. (2016). Efecto de la práctica deportiva en la relación entre las habilidades motoras, el autoconcepto físico y el autoconcepto multidimensional. Revista de Psicología del Deporte, 25(1), 19-25.

Ortega, E. (2005). Autoeficacia y deporte. Sevilla: Wanceulem.

Pastor, Y., \& Balaguer, I. (2001). Relaciones entre autoconcepto, deporte y competición deportiva en los adolescentes valencianos. Psicologiaonline. Recuperado de http:// www.psicologia-online.com

Pastor, Y., Balaguer, I., \& García-Merita, M.L. (2003). El autoconcepto y la autoestima en la adolescencia media: análisis diferencial por curso y género. Revista de Psicología Social, 18(2), 141-159. https://doi. org/10.1174/021347403321645258

Penado, M., \& Rodicio-García, M. L. (2017). Análisis del autoconcepto en las víctimas de violencia de género entre adolescentes. Suma psicológica, 2(4), 107-114. https://doi.org/10.1016/j.sumpsi.2017.08.001

Pérez, S. M., García, C. G., \& Ferriol, Á. G. (2015). Efecto de la práctica deportiva en la relación entre las habilidades motoras, el autoconcepto físico y el autoconcepto multidimensional. Revista de Psicología del Deporte, 25(1), 19-25.
Pezzuti, T., Pirouz, D., \& Pechmann, C. (2015). The effects of advertising models for age-restricted products and self-concept discrepancy on advertising outcomes among young adolescents. Journal of Consumer Psychology, 25(3), 519-529. doi:http:// dx.doi.org/10.1016/j. jcps.2015.01.009

Piéron, M. (2002). Estudi sobre els hàbits esportius dels escolars d'Andorra. Andorra: Ministeri d'Educació, Joventut i Esports, Govern d'Andorra.

Rangel, Y.S., Mayorga, D., Peinado, J.E., \& Barrón, J.C. (2017). Actividad física, autoconcepto físico y bienestar psicológico en estudiantes universitarias mexicanas Revista de Psicología del Deporte,26(Suppl 2), 61-69

Reigal, R., Videra, A., Parra, J. L., \& Juárez, R. (2012). Actividad físico deportiva, autoconcepto físico y bienestar psicológico en la adolescencia. Retos: Nuevas tendencias en Educación Física, Deporte y Recreación 22(1), 19-23.

Rodríguez, A., \& Fernández, A. (2005). Los componentes del bienestar psicológico y el autoconcepto físico de los adolescentes. En Fajardo, M. I., Vicente, F., Ventura, A., Ruiz, I., y del Barrio, J. A. (Eds.), Aportaciones psicológicas y mundo actual. Dando respuestas (pp. 465-480). Badajoz: Psicoex.

Rodríguez-García, P.L., Gálvez, A., García-Cantó, E., Pérez-Soto, J.J., Rosa, A., Tárraga... Tárraga, P.L. (2015). Relationship between the Self-Concept and Muscular Strength in Southern Spanish Children. J Psychol Psychother 5, 222.

Rodríguez-García, P.L., Tárraga, L., Rosa, A., García-Cantó, E., PérezSoto, J.J., Gálvez, A., \& Tárraga, P. (2014). Physical Fitness Level and Its Relationship with Self-Concept in School Children. Psychology, 5, 2009-2017.

Rosa, A. (2015). Niveles de condición física y su relación con el perfil de autoconcepto en escolares de 8 a 11 años de la región de Murcia. EBalonmano.com: Revista de Ciencias del Deporte, 11(3), 228-229.

Rosa, A., García, E., \& Carrillo, P.J. (2019). Actividad física, condición física y autoconcepto en escolares de 8 a 12 años. Retos, 35, 236-241.

Sánchez-Alcaraz, B. J., \& Gómez-Marmol, A. (2014). Autoconcepto físico en una muestra de estudiantes de primaria y su relación con el género y la práctica deportiva extraescolar. e-balonmano.com: Revista de Ciencias del Deporte, 10(2), 113-120.

Salum-Fares, A., Reséndiz, E., \& Saldivar, H. (2012) Diferencias del autoconcepto en estudiantes de escuelas secundarias públicas y privadas de ciudad de Victoria, Tumaulipas, México. Revista Electrónica de Psicología Iztacala, 15(2), 372-393.

Taylor, A., \& Fox, K. (2005). Effectiveness of a primary care exercise referral intervention for changing physical self-perceptions over nine months. Health Psychology, 24(1), 11-21. https://doi. org/10.1037/0278-6133.24.1.11

Thomson, J. K., Penner, L. A., \& Atabe, M. N. (1990). Procedures, problems, and progress in the assessment of body images. En T. F. Cash, y T. Pruzinsky (Eds.), Body images: Development, deviance, and change. New York: Guilford.

Topcu, S., Orthon, F. S., Tayfun, M., Ucaktuerk, S. A., \& Demirel, F. (2016). Anxiety, depression and self-esteem levels in obese children: a case-control study. Journal of Pediatric Endocrinology \& Metabolism, 29(3), 357-361. doi:10.1515/JPEM-2015-0254

Trautwein, U., Gerlach, E., \& Lüdtke, O. (2008). Athletic classmates, physical self-concept, and free-time physical activity: A longitudinal study of frame of reference effects. Journal of Educational Psychology, 100(4), 988-1001. https://doi.org/10.1037/0022-0663.100.4.988

Videra, A., \& Reigal, R. (2013). Autoconcepto físico, percepción de salud y satisfacción vital en una muestra de adolescentes. Anales de psicología, 29(1), 141-147. https://doi.org/10.6018/analesps.29.1.132401 